\title{
L'éthique et la standardisation des pratiques enseignantes
}

Denis Jeffrey Université Laval
Ethics and standardization of teaching practices

\section{R ésumé}

L'éthique est une préoccupation professionnelle fondamentale. Tout professionnel se distingue habituellement par une éthique qu'il partage avec ses pairs. $0 r$, les enseignants québécois ne se sont jamais agrégés autour de normes éthiques communes. II semble même qu'à défaut d'une éthique professionnelle commune prévaut encore au sein de leur profession une morale vertuiste héritée des anciennes formations des maîtres. Nous soutenons ici que cette ancienne morale

est dépassée pour évaluer le travail des enseignants et qu'il serait préférable qu'ils se donnent une éthique professionnelle fondée sur la responsabilité des actes.

Dans ce texte, nous présentons d'abord quelques impasses dans le processus de professionnalisation des pratiques enseignantes. Par la suite, nous examinons des considérations historiques pour saisir pourquoi le vertuisme religieux s'impose encore aujourd'hui pour juger de la moralité d'un enseignant. Nous terminons avec des arguments pour soutenir une éthique de la responsabilité pour les enseignants québécois.

Éthique, éthique professionnelle, professionnalisation, savoirs professionnels, enseignants, enseignements

\section{Abstract}

Ethics is a fundamental professional concern. Any professional is usually characterized by an ethic he shares with his peers. Or, Québec teachers are never aggregated to around common ethical standards. It even seems that without a common ethic, still prevails within their profession a «moral virtuism» inherited from ancien teachers training. We argue here that we connot used this old morality for evaluating the work

of teachers and it would be better that they give to themselves a professional ethic of responsibility. In this paper, we first present some impasses in the process of professionalisation of teaching practices. Subsequently, we examine historical considerations to understand why the religious virtuism is still needed today to judge the morality of a teacher. We end with arguments to support an ethic of responsibility for Quebec teachers. doi :10.18162/fp.2016.351

\section{Introduction}

L'éthique est une préoccupation professionnelle fondamentale. Comme l'ont souligné Gohier, Anadón, Bouchard, Charbonneau et Chevrier (1999) et Legault (2003a), tout professionnel se distingue par une éthique qu'il partage avec ses pairs. Une éthique professionnelle est constituée par "l'ensemble des règles de bonne conduite dont une profession se dote pour régir son fonctionnement au regard de sa mission "(Verdier, 1999, p. 19). Ces règles ne sont pas que "morales", elles sont également sociales, politiques et juridiques.

AuQuébec, deuxconceptions del'éthique foncièrementcontrastées régulent les pratiques enseignantes. La première est issue du vertuisme religieux hérité de la formation des maîtres offerte dans les anciennes écoles normales tenues par des religieuses. Avant les années 1960 au Québec ${ }^{1}$, une femme pouvait être chassée de l'enseignement du seul fait qu'elle se mariait. On considérait alors qu'elle ne pouvait plus, ayant perdu sa chasteté, être un modèle de moralité. La seconde s'appuie sur une normativité professionnelle et interpelle le sens des responsabilités des enseignants. Soulignons d'entrée de jeu que les enseignants québécois ne se sont pas encore dotés d'un code d'éthique. Les normes éthiques qui régissent leur profession proviennent essentiellement de la Loi de l'instruction publique (LIP), de diverses législations provinciales et de la jurisprudence des tribunaux.

Les enseignants québécois subissent une immense pression pour adopter un comportement moral exemplaire, lequel n'est pas toujours bien différencié du vertuisme des anciennes formations religieuses. Aussi, dans trois importantes décisions juridiques qui datent de 1996, la Cour suprême du Canada référait directement au vertuisme religieux pour exiger des enseignants un comportement 
irréprochable dans la classe et hors de la classe pendantles 365 jours de l'année(Jeffrey, Harvengt, Vachon et Deschesnes, 2008). Cela signifie qu'on leur demande de conserver leur statut d'enseignant même dans leurs activités privées. Ces décisions servent encore aujourd'hui d'arguments pour blâmer un enseignant qui n'aurait pas un comportement considéré exemplaire. Or, c'est notre hypothèse de recherche, ce recours au vertuisme religieux hérité des écoles normales freine la professionnalisation des enseignants.

Dans ce texte, nous présentons d'abord, en prolégomènes, quelques impasses dans le processus de professionnalisation des pratiques enseignantes. Par la suite, nous examinons des considérations historiques pour saisir pourquoi le vertuisme religieux s'impose encore aujourd'hui pour juger de la moralité d'un enseignant. Nous terminons avec des arguments pour soutenir une éthique de la responsabilité pour les enseignants québécois. Il n'est pas déraisonnable de penser que le processus de professionnalisation des enseignants et de leurs pratiques les amène à développer une éthique de la responsabilité.

\section{Professionnaliser les pratiques enseignantes}

La question de la professionnalisation des enseignants n'est pas nouvelle. En 1963, les signataires du rapport Parent souhaitent qu'ils deviennent de véritables professionnels : "En situant la formation des instituteurs au niveau universitaire, nous avons recommandé la première mesure requise pour que l'enseignement puisse se comparer aux autres professions » (Parent, 1963, Tome 2, p. 213). Ils sont unanimes à recommander que la formation des maîtres soit transférée dans les universités. C'est dans cet esprit que les facultés des sciences de l'éducation québécoises du secteur francophone voient le jour au milieu des années 1960. Gohier (1997) et Tardif, Lessard et Gauthier (1998) considèrent, à cet égard, que la formation universitaire a contribué, au cours des cinquante dernières années, à la professionnalisation de l'enseignement et des enseignants.

Depuis 2001, les nouveaux programmes universitaires de formation à l'enseignement sont fondés sur un référentiel de compétences qui propose explicitement la professionnalisation des enseignants. Ces

programmes comportent deux orientations. La première vise à former un professionnel apte à exercer des compétences d'une manière responsable. La seconde propose de former un pédagogue cultivé, c'est-à-dire qui maîtrise un vaste ensemble de contenus culturels afin de rendre la matière à enseigner plus significative. Ces deux orientations sont traduites en douze compétences professionnelles. 


\section{Tableau 1}

Référentiel des 12 compétences pour la formation des maîtres (Ministère de l'Éducation, du Loisir et du Sport, 2001)

\begin{tabular}{|c|c|}
\hline $\begin{array}{l}\text { Fondements du métier } \\
\text { 1. Compétence disciplinaire et culturelle } \\
\text { 2. Compétence dans la langue d'enseignement }\end{array}$ & $\begin{array}{l}\text { Contexte social et scolaire } \\
\text { 7. Compétence à adapter l'enseignement aux besoins des } \\
\text { catégories particulières d'élèves } \\
\text { 8. Compétence à intégrer les TIC } \\
\text { 9. Compétence à coopérer avec les partenaires } \\
\text { 10. Compétence à travailler en équipe }\end{array}$ \\
\hline $\begin{array}{l}\text { Acte d'enseigner } \\
\text { 3. Compétence à planifier des situations d'enseignement et } \\
\text { d'apprentissage } \\
\text { 4. Compétence à piloter des situations d'enseignement et } \\
\text { d'apprentissage } \\
\text { 5. Compétence à évaluer des situations d'enseignement et } \\
\text { d'apprentissage } \\
\text { 6. Compétence à gérer la classe }\end{array}$ & $\begin{array}{l}\text { Identité professionnelle } \\
\text { 11. Compétence à s'engager dans une démarche de dévelop- } \\
\text { pement professionnel } \\
\text { 12. Compétence à agir de façon éthique }\end{array}$ \\
\hline
\end{tabular}

Ce référentiel répond aux critères habituellement retenus pour définir une profession autonome : une formation plutôt longue et spécialisée (quatre années au Québec), des contenus pratiques et théoriques reconnus et une activité intellectuelle qui engage la responsabilité individuelle (Hensler, 2004, p. 186; Lemosse, 1989, p. 57). Plusieurs autres critères peuvent être évoqués pour déterminer si l'enseignement est une profession : 1) un service essentiel et unique pour la société, 2) une formation spécialisée qui mène à une certification et donne un droit de pratique exclusif, 3) des savoirs spécialisés exclusifs, 4) une pratique qui demande une grande autonomie intellectuelle, 5) des finalités qui lui sont propres, 6) une mise à jour constante des connaissances pour améliorer la pratique,7) de grandes responsabilités morales vis-à-vis des élèves, des pairs, des parents et de la population, 8) des comités de pairs qui réfléchissent sur les pratiques de la profession, sa promotion, son image professionnelle et ses balises éthiques (Anadón, 1999).

Un professionnel n'a pas une pratique routinière ou mécanique, et on le sait capable de réfléchir sur tous les aspects de son travail (Schön, 1994). Habituellement, les professionnels constituent des associations ou des ordres pour définir leurs pratiques, pour baliser les formations initiale et continue, pour délimiter les actes qu'ils peuvent poser et pour promouvoir leurs idéaux. Aussi, ils prennent en charge la régulation et la supervision du travail de leurs pairs (Bourdoncle, 1998). Cela suppose, comme le relevait Perrenoud (1994), une capacité collective de s'organiser, de développer les différentes dimensions de la profession et de représenter les membres. Nous pourrions ajouter d'autres critères comme celui de se doter d'un comité disciplinaire qui a le mandat de traiter les fautes et manquements professionnels.

Tous ces critères visent à démarquer les professionnels de ceux qui n'en sont pas et contribuent à mieux établir les actes qui leur appartiennent. Par conséquent, ils définissent le niveau de professionnalité ${ }^{3}$ qui confère le statut de professionnels. Il est possible de regrouper tous les critères qui déterminent la 
professionnalité sous trois aspects : 1) le développement du statut professionnel (identité, autonomie, autorité) et de la reconnaissance sociale de la profession, 2) la détermination et la standardisation des savoirs, des pratiques et des formations, et 3) le professionnalisme et l'éthique. Pour éviter de les confondre, il apparaît prudent de distinguer les concepts de professionnalité, de professionnalisme et de professionnalisation. La professionnalité renvoie aux critères qui déterminent le passage d'un métier à une profession. Le professionnalisme permet de juger de la qualité d'une pratique accomplie dans le respect des personnes qui reçoivent les services et dans le respect de l'art de faire. La professionnalisation concerne d'une part, le rehaussement de l'identité et du statut des enseignants, et d'autre part, la standardisation ou la normalisation des savoirs, des pratiques et des formations. Par conséquent, la professionnalisation des enseignants et la professionnalisation de l'enseignement sont indissociables, mais distinctes. Même si l'enseignement n'est pas reconnu comme une profession dans le sens de la loi québécoise, et même si les enseignants ne sont pas regroupés dans un ordre professionnel, leur formation les prépare à se comporter comme des professionnels et leurs syndicats défendent l'idée qu'ils sont des professionnels ${ }^{4}$.

Cela dit, l'enseignement peut-il être considéré comme une profession au Québec? Et les enseignants sont-ils des professionnels? Plusieurs auteurs, à l'instar de Gohier (2013), soutiennent que les enseignants sont des professionnels, même si ce statut n'est pas accordé par un ordre professionnel, même s'ils n'ont pas de droit de regard sur la formation et l'accréditation des membres, et même s'ils n'ont pas un code d'éthique pour soutenir leur identité professionnelle. Sans avoir obtenu une reconnaissance formelle, souligne-t-elle, les enseignants doivent se comporter comme des professionnels. Dès lors, nous devrions supposer qu'ils sont capables de professionnalisme et que leurs savoirs, leurs pratiques et leur formation initiale et continue répondent aux normes les plus exigeantes d'une profession.

À bien des égards, et c'est la position défendue ici, la professionnalisation de l'enseignement et des enseignants devrait conduire à la standardisation des savoirs et des pratiques enseignantes et à l'établissement d'une éthique de responsabilité qui les rend imputables de leurs actes et décisions. Plutôt que de parler de standardisation des pratiques, certains auteurs préfèrent le terme d'« institutionnalisation» des pratiques (Sorel et Wittorski, 2005). Nous conserverons le terme de standardisation, même s'il soulève certains malaises liés à la technicisation des actes professionnels. Nous n'aborderons pas cette question qui mérite à elle seule une longue explication. Toutefois, retenons que l'un et l'autre terme impliquent des actes que seul le professionnel reconnu compétent peut exercer et en assumer la responsabilité.

Dans la perspective que nous développons ici, une pratique est standardisée dans la mesure où elle répond à ces trois critères : 1) elle est définie à partir de données probantes issues de l'expérience et de la recherche savante, 2) elle est réservée aux seuls professionnels formés et compétents, 3) le professionnel est entièrement responsable de ses pratiques. Dans cette optique, l'enseignant devrait être capable de justifier ses actes et ses décisions sur des expériences qui ont fait leur preuve et sur des travaux savants reconnus, partagés, discutés par les pairs et régulièrement révisés et adaptés aux nouvelles données de la recherche. L'éthique professionnelle, dans l'optique d'une éthique de responsabilité que nous défendons, exige des enseignants de justifier leur travail. Or, il va de soi qu'il sera plus facile pour les enseignants de justifier leur travail sur des standards professionnels, d'où l'importance de la standardisation des pratiques enseignantes. 


\section{La standardisation des pratiques enseignantes}

Nous allons présenter une situation d'interaction entre enseignants et élèves pour laquelle la standardisation d'une pratique pédagogique est devenue incontournable dans le contexte scolaire contemporain. Cette situation est tirée des recherches que nous menons sur l'examen des jugements des cours canadiennes dans des causes où un enseignant est accusé pour une faute professionnelle (Jeffrey et al., 2008). Nous avons découvert, en analysant les décisions des tribunaux concernant des enseignants accusés de voies de fait, que ces derniers ne connaissaient pas les procédures normatives éthiques et pédagogiques pour mener avec compétence une intervention physique auprès d'un élève. Dans la majorité de ces causes, l'enseignant accusé avait immobilisé un élève, l'avait retenu en lui saisissant un bras ou l'avait déplacé en le soulevant. Dans quelques causes plutôt rares, l'enseignant avait frappé un élève avec la main ou le pied. Pour ces dernières situations, un enseignant pourrait sembler fautif puisqu'il aurait maltraité un élève. Toutefois, chaque situation doit être examinée dans sa particularité. En fait, on ne peut présumer de la culpabilité d'un enseignant. Parmi ceux qui ont volontairement frappé un élève, certains ont été acquittés, d'autres ont été condamnés. Dans les autres situations, l'intervention physique de l'enseignant visait à sécuriser un élève ou à protéger les autres élèves, à l'empêcher de commettre un délit comme casser ou lancer un objet, à le retenir afin qu'il ne bouscule pas ou ne frappe pas un autre élève, à l'immobiliser alors qu'il sortait de la classe sans permission, etc. Il aurait été préférable que les enseignants s'abstiennent de mener une intervention physique auprès d'un élève, mais les cas de nécessité ne sont pas rares ${ }^{5}$, même au niveau primaire où les accusations sont aussi nombreuses qu'au niveau secondaire.

Les standards de l'intervention physique pour immobiliser ou pour retenir un élève ne sont pas connus des enseignants, à l'instar de toutes les autres interventions qui concernent un contact physique avec un élève pour le motiver, l'aider à se vêtir, le consoler ou pour modeler un geste d'apprentissage. Rappelons qu'une telle intervention acquiert la valeur d'un acte professionnel parce qu'elle est validée par des expériences qui ont fait leur preuve, des normes juridiques et éthiques et les recherches savantes portant sur ce sujet ${ }^{6}$. En fait, une pratique enseignante n'est pas standardisée tant que ses finalités, ses conditions d'accomplissement et les procédures à suivre nont pas été définies et explicitées sur la base de connaissances reconnues et validées.

Dans plusieurs accusations pour voies de fait, les enseignants avaient agi d'une manière spontanée et personnelle pour contenir ou maintenir un élève. Ils ne pensaient pas que leur intervention pouvait être préjudiciable. Dans la cause Gonthier (2008), l'enseignant a été poursuivi pour voies de fait parce qu'il avait saisi par le bras un élève qui cherchait à sortir de la classe quelques minutes avant la fin du cours. Cet enseignant agissait alors pour le bien de l'élève. Or, c'est là que le bât blesse, la même intervention menée selon les normes établies l'aurait mieux protégé contre une accusation criminelle. Cet enseignant a eu gain de cause à la suite d'une bataille judiciaire pénible et déshonorante. Par contre, il n'est pas revenu à l'enseignement, brisé par de telles accusations et incapable d'affronter avec confiance une classe d'élèves. En effet, une voie de fait est une accusation criminelle grave qui n'est jamais traitée à la légère. Retenons de cette poursuite contre l'enseignant Gonthier que ce dernier ne savait pas qu'il pouvait commettre une erreur professionnelle lorsqu'il a immobilisé un élève. 
En réponse à un grand nombre d'accusations pour voies de fait, plusieurs syndicats d'enseignants du Québec ont conseillé à leurs membres d'éviter tout contact physique avec les élèves. Cette position n'est pas acceptable. Il appert plutôt que la meilleure solution pour les enseignants consiste à mener une intervention physique à l'aune des standards professionnels qui visent autant la protection des élèves que des enseignants. En fait, toutes pratiques enseignantes qui impliquent de toucher un élève, comme toutes les autres pratiques enseignantes par ailleurs, ne peuvent être improvisées. C'est pourquoi nous insistons sur la standardisation des pratiques enseignantes.

Dans la réalité de la classe, la plupart des enseignants ne savent pas s'ils peuvent intervenir physiquement auprès d'un élève pour l'immobiliser ou pour l'empêcher de commettre un délit. Au demeurant, ils ne savent pas s'il leur revient ou non d'intervenir physiquement dans telle ou telle situation d'interactions avec un élève. Par ailleurs, ils ne sauraient comment mener une intervention physique sans violer les lois canadiennes et les normes éthiques fondamentales ${ }^{7}$. Si les standards d'une intervention physique étaient connus, cela aurait pour effet de rassurer les enseignants quant à leur niveau de compétence. De plus, cela leur permettrait de montrer qu'ils agissent en professionnel et d'assumer au point de vue éthique l'entière responsabilité de l'intervention. Il va de soi que l'adoption d'une conduite standardisée contribue également à conserver la confiance des élèves, des parents et à garantir leur travail auprès de la population. Dès lors, l'intervention physique pourrait être évaluée à l'aune d'une normativité professionnelle.

Alors que la plupart des actes des médecins, des infirmiers, des psychologues et des autres professionnels ont été standardisés, nous constatons les réticences des enseignants pour établir des standards professionnels. Par conséquent, il est pertinent de s'intéresser à ces réticences et de poursuivre les recherches qui contribuent à définir des standards et des normes pour baliser les pratiques pédagogiques et éthiques des enseignants.

$\mathrm{Au}$ terme de cette partie, demandons-nous si les enseignants peuvent être considérés comme des professionnels. Force est de constater qu'au Québec les enseignants ne forment pas, au point de vue de la loi, un véritable corps professionnel. Même s'ils reçoivent une formation professionnelle, même s'ils font preuve de professionnalisme et se considèrent eux-mêmes comme des professionnels, ils ne partagent pas le même statut que les professionnels reconnus par l'Office des professions du Québec. Aussi, leur identité sociale comme professionnels est loin dêtre reconnue par la population. On doit par contre considérer que la standardisation de leurs pratiques, notamment d'un point de vue éthique, pourrait contribuer à rehausser leur image de marque.

Nonobstant ces remarques, le processus de professionnalisation des enseignants a gagné quelques échelons au Québec lorsque la formation à l'enseignement, en 2001, est devenue officiellement une formation professionnelle. Cela a pu motiver plusieurs enseignants à réfléchir sur ce que signifie être un professionnel. De son côté, Gohier $(1997,2007)$ et Gohier et al. (1999) ont montré que les enseignants sont en train de se construire, depuis les années 1970, une identité en phase avec l'idéal de professionnalisation. Toutefois, adopter une attitude professionnelle et partager avec ses pairs une normativité professionnelle établie sur une base de connaissances reconnues sont deux choses distinctes. Un enseignant peut montrer une attitude professionnelle sans toutefois adhérer à des standards professionnels communs à tous les enseignants. Cela signifie que la professionnalisation des enseignants (de leur statut) devance grandement la professionnalisation de l'enseignement. Nous 
pouvons penser que la standardisation des pratiques enseignantes pourra mener, à long terme, à délimiter les actes qui appartiennent aux enseignants et ceux qui ne lui appartiennent pas. En fait, la question des actes réservés est loin dêtre résolu en enseignement puisque les commissions scolaires ouvrent leurs classes à des personnes qui ne sont pas formées en enseignement, ou bien encore elles exigent d'un nouvel enseignant qu'il enseigne des matières pour lesquelles il n'est pas formé ${ }^{8}$. Même si la formation à l'enseignement fut transférée dans les universités, les processus de professionnalisation de l'enseignement et des enseignants sont loin d'être achevés.

\section{Professionnalisation et normes éthiques communes}

La reconnaissance d'une profession est d'autant mieux acquise si les normes éthiques sont clairement établies. Des normes, pour la profession enseignante, qui placeraient la protection et la réussite des élèves au-dessus de tout ${ }^{9}$. Si les questions éthiques dominent le champ des professionnels, c'est parce qu'une autonomie accrue leur est reconnue (Legault, 2003b). Dubar (2000) souligne à ce propos que l'autonomie du professionnel constitue la principale raison pour laquelle existe un code de déontologie dans les professions libérales. Étant entièrement redevable de ses actes, le professionnel doit montrer sa volonté d'agir d'une manière responsable en adhérant aux standards de sa pratique, mais aussi aux normes éthiques partagées par tous les professionnels de son groupe.

$\mathrm{Au}$ Québec, les enseignants ne sont pas encore liés par un code d'éthique (ou de déontologie). Dans les établissements scolaires, ils doivent parfois adhérer à des règles internes écrites et non écrites (comme le code de vie de l'école qui touche notamment les comportements vestimentaires et les activités de la vie privée) qui les amènent à adopter des attitudes et des comportements qui contreviennent parfois à leurs propres normes et valeurs ${ }^{10}$. Ces règles n'ont pas été produites exclusivement pour les enseignants, c'est pourquoi les enseignants ont raison de s'en méfier, d'autant plus qu'elles ne reconnaissent pas les particularités de leur autonomie et leur autorité professionnelles.

Dans cette partie de notre texte, nous discutons de la nécessité de l'établissement de normes éthiques pour la profession enseignante. Nous soutenons qu'une éthique de la responsabilité est appelée à remplacer le vertuisme religieux auquel s'associent encore aujourd'hui nombre d'enseignants. Nous allons d'abord présenter le vertuisme religieux afin d'en expliquer la perdurance.

Le vertuisme religieux fut imposé aux enseignants par l'Église catholique québécoise qui, jusqu'au milieu des années 1960, contrôlait le système scolaire (Hamel, 1995; Tardif, 2013). Pour obtenir un brevet d'enseignement qui leur permettait d'enseigner les six premières années du primaire dans une petite école de rang, les institutrices devaient obtenir un certificat de moralité de leur curé :

Dès les premières lois scolaires, il était prévu que les enseignants produisent un certificat de moralité signé par le curé ou le pasteur protestant et par trois commissaires de leur localité d'origine, au moment de leur embauche, et plus tard devant le bureau d'examinateurs qui devait évaluer leur compétence. (Charland, 2000, p. 328) 
Leur moralité devait être irréprochable et exemplaire en toutes circonstances. Maurice Tardif écrit à ce sujet :

Avant d'être une fonction savante, une occupation intellectuelle basée sur l'acquisition et la transmission de connaissances, l'enseignement apparaît au XIX ${ }^{\mathrm{e}}$ siècle (et durant une bonne partie du $\mathrm{XX}^{\mathrm{e}}$ siècle également) d'abord et avant tout comme un « métier moral » : le « savoir-enseigner» ou, si l'on préfère, la pédagogie se fonde et se légitime sur le savoir-être de l'enseignant, ce dernier étant régi par les règles de bonne conduite (sobriété, respect des autorités, etc.) et profondément imprégné de vertus religieuses. (2005, p. 24)

En fait, dans le Québec catholique d'autrefois, les instituteurs et les institutrices devaient être des modèles de moralité. Dans les écoles de formation des maîtres de l'époque, ils apprenaient à se plier aux caprices des communautés religieuses. Le climat académique dans ces écoles était calqué sur celui des couvents. La discipline était sévère. Les futures enseignantes ne pouvaient se laisser distraire par des affaires de cœur. Elles devaient incarner un idéal religieux de moralité auprès des élèves et de leurs parents. Les jeunes filles s'engageaient dans l'enseignement pour être des éducatrices du peuple.

Une moralité plus élevée était demandée aux jeunes filles du fait, pensait-on alors, qu'elles se laissaient plus facilement séduire par tous les serpents de ce monde. Les autorités scolaires étaient également plus sévères à leur égard. Leur identité de femme les déclassait. Dès qu'elles prenaient époux, elles étaient évincées de l'école. Une femme ne pouvait être à la fois mère et enseignante.

$\mathrm{Au}$ cours de leur formation, elles devaient mémoriser les leçons de religion. Peu de temps était alors consacré à la pédagogie. Bourdoncle $(1990,1991)$ souligne que ces femmes qui entraient dans l'enseignement provenaient du petit peuple. On les préparait à s'élever au-dessus de leur milieu social afin d'être un modèle moral auprès des leurs. Dans les représentations sociales de cette époque, un bon modèle moral incarnait les vertus religieuses du catholicisme. En fait, former une future enseignante avant les années 1970 revenait à la soumettre à une éducation morale rigoureuse. Les hommes subissaient le même type de formation, mais la sévérité était moindre à leur égard puisque la théologie soutenait qu'ils étaient plus proches, du fait de leur raison, du divin. Cette conception moralisatrice du travail enseignant était enchâssée dans les mœurs d'une population canadienne-française qui voyait dans un enseignant une personne dont la moralité devait être irréprochable.

C'est sur ce même vertuisme religieux qu'en 1996 les juges de la Cour suprême du Canada ont justifié plusieurs décisions dans diverses causes qui touchent les enseignants (Jeffrey et al., 2008). À cet égard, maître Linda Lavoie, spécialiste en droit scolaire, affirme que les juges de la plus haute cour du pays entretiennent une conception rétrograde de la moralité des enseignants. Pour les juges, souligne-telle, l'enseignant devrait incarner «le rôle de modèle de moralité qu'incarnaient autrefois les nobles du village. Il devrait donc accéder à une hauteur morale plus élevée que celle d'un médecin, d'un notaire, d'un curé ou d'un avocat » (2001). En fait, en fondant leurs décisions sur ce vertuisme religieux, la Cour suprême a contribué à entretenir un moralisme désuet qui freine la professionnalisation de l'enseignement. En effet, les arguments de la Cour suprême influencent les décisions des autres tribunaux et des commissions scolaires qui peuvent en tout temps s'y référer.

Cette conception vertuiste de la morale de l'enseignant domine encore aujourd'hui le champ éducatif. Des formateurs universitaires et des directions scolaires, sans en avoir toujours conscience, en font la promotion à travers leurs attentes morales à l'égard des enseignants. Par ailleurs, plusieurs 
enseignants continuent de croire erronément qu'ils doivent incarner les vertus religieuses d'autrefois. Ils se perçoivent comme des modèles de moralité sur lesquels les élèves doivent se mirer. À bien des égards, cette conception ancienne de l'ethique enseignante perdure malgré le développement au cours des dernières décennies d'une orientation éthique professionnelle inscrite notamment dans le référentiel de compétences de 2001 et dans la LIP.

Quelle est cette éthique professionnelle qui devrait remplacer le vertuisme religieux? Quels sont ses fondements? Nous allons répondre à ces deux questions en mettant en contraste le vertuisme religieux et l'éthique professionnelle des enseignants, nommée ici éthique de la responsabilité. La douzième compétence du référentiel de compétence présenté plus haut dans ce texte est dédiée à la dimension éthique de l'enseignement. Son contenu touche l'ensemble des responsabilités que les enseignants doivent assumer dans le cadre de leurs fonctions. Elle s'inscrit explicitement dans le giron des onzième et douzième compétences, c'est-à-dire dans une logique de professionnalisation de l'enseignement. Nos travaux antérieurs nous amènent à considérer qu'une éthique de la responsabilité peut être fondée sur ces quatre principes : 1) l'autonomie accrue des enseignants; 2) leurs nombreuses responsabilités à l'égard des élèves et du système éducatif; 3) la reconnaissance de leur autorité professionnelle et 4) leur capacité à conserver la confiance des élèves et du public. Ces principes ne sont pas exclusifs l'un de l'autre, mais s'interpénètrent et se complètent.

Dans le cadre d'une éthique de la responsabilité, les enseignants doivent être capables de justifier leurs actes et leurs décisions à l'aune des connaissances établies de leur profession. Il leur est donc demandé de pouvoir expliquer clairement et raisonnablement leurs pratiques pédagogiques. Cela signifie, en réalité, qu'un enseignant doit être capable de faire comprendre aux élèves, aux parents, aux membres de la direction comme à toute autre personne du public pourquoi il choisit telle activité pédagogique plutôt qu'une autre, pourquoi il intervient en gestion de classe de telle manière plutôt que d'une autre et pourquoi il évalue les productions des élèves à partir de tels ou tels outils de correction plutôt qu'avec tels autres outils. Il doit donc être capable de porter un éclairage professionnel sur tous ses actes et sur toutes ses décisions. En fait, la responsabilité appelle les enseignants à s'engager à agir en tout temps avec compétence. Un professionnel compétent, en somme, est capable d'expliquer tout ce qu'il fait à l'aune de savoirs établis ${ }^{11}$.

Contrairement au vertuisme religieux, l'éthique de la responsabilité n'est pas liée à une disposition morale de la personne ou à une capacité de composer une image vertueuse de soi. Elle touche plutôt à la capacité de pratiquer l'enseignement en phase avec les standards de la profession. On comprend alors que le vertuisme religieux, fondé sur les qualités morales de l'enseignant, ne les engage pas en termes de maîtrise des compétences professionnelles. Comme l'avait souligné Lucien Morin, « les qualités morales qui font d'un individu une "bonne" personne ne sont pas nécessairement les mêmes qui font de lui un "bon" professionnel» (1997, p.122). Ces deux formes de qualités morales ne sont pas antinomiques, mais elles doivent être distinguées. Elles font appel à deux modèles de moralité qui ont chacune leur finalité propre.

Une éthique de la responsabilité doit être fondée sur des attentes professionnelles, et non pas sur des attentes en termes de vertus. Un enseignant peut manquer de vertu - par exemple montrer moins de sollicitude ou de bienveillance qu'un autre - mais être compétent dans ses tâches professionnelles. D’un point de vue moral et juridique, le manque de vertu ne peut être évalué avec les mêmes critères que le manque de compétence. En fait, le manque de vertu n’induit pas derechef une faute professionnelle. Il 
faudrait montrer d'une manière raisonnable que le manque de vertu devient une entrave pour mener des tâches enseignantes avec compétence. Soulignons qu'une éthique professionnelle propose des normes et des critères d'intégrité pour évaluer un manquement moral de la part d'un enseignant. C'est à l'aune d'une norme éthique d'intégrité professionnelle que devrait être examiné le comportement d'un enseignant qui, notamment, consomme des drogues ou pratique le naturisme.

Dans le cadre des recherches que nous menons depuis plus de dix ans sur les fautes professionnelles commises par des enseignants québécois (Jeffrey, 2013, 2014, 2015; Jeffrey et al., 2008, ), nous avons observé que la majorité des arguments de commissions scolaires sont fondés sur le vertuisme religieux. Or, se demander si un enseignant est vertueux ou se demander si un enseignant est capable d'assumer ses responsabilités avec professionnalisme ne participe pas du même modèle normatif. Dans la première optique, on s'intéresse aux qualités morales de la personne de l'enseignant, dans la seconde on s'intéresse aux compétences professionnelles de l'enseignant. Elles peuvent se chevaucher, mais le professionnalisme doit primer le vertuisme.

\section{Conclusion}

Une majorité d'enseignants acceptent le vertuisme religieux par tradition ou par ignorance qu'il pourrait se regrouper autour de véritables normes éthiques. Il devient donc judicieux de faire la promotion d'une éthique de la responsabilité en montrant sa pertinence et en encourageant les futurs maîtres et l'ensemble du corps enseignant à s'y intéresser. Nous pouvons supposer qu'une normativité éthique professionnelle s'imposera avec la professionnalisation des enseignants et de leurs pratiques du fait que ces derniers seront alors appelés à justifier chacune de leurs actions et chacune de leurs décisions. En fait, la professionnalisation des pratiques permettra de mieux identifier leurs responsabilités et les limites de leur autonomie. Les enseignants pourront connaître les pratiques qu'ils sont autorisés à poser, puisque chacune de ces pratiques exige une formation qualifiante préalable (Legault, 2003a, p. 35). Il sera alors possible de protéger et de réserver les actes que les enseignants peuvent poser. $\mathrm{Au}$ final, la professionnalisation des pratiques enseignantes permettra de mieux identifier qui peut enseigner, de reconnaître les bonnes pratiques et de déterminer les responsabilités communes à tous les enseignants.

\section{Références}

Anadón, M. (1999). L'enseignement en voie de professionnalisation. Dans C. Gohier, N. Bednarz, L. Gaudreau, R. Pallascio et G. Parent (dir.). L'enseignant, un professionnel (p. 1-20). Québec, QC : Presses de l'Université du Québec.

Anderson, E. M. et Levine, M. (1999). Concerns about allegations of child sexual abuse against teachers and the teaching environment. Child Abuse E' Neglect, 23(8), 833-843. http://dx.doi.org/10.1016/s0145-2134(99)00054-x

Bourdoncle, R. (1990). De l'instituteur à l'expert : les IUFM et l'évolution des institutions de formation. Recherche et formation, (8), 57-72.

Bourdoncle, R. (1991). La professionnalisation des enseignants : analyses sociologiques anglaises et américaines. Revue française de pédagogie, 94(1), 73-91. http://dx.doi.org/10.3406/rfp.1991.1368

Bourdoncle, R. (1998). Artisan moral ou professionnel? La place des valeurs dans la conception de la fonction et de la formation des enseignants. SPIRALE - Revue de recherches en éducation, (21), 25-34. Repéré à http://spirale-edu-revue.fr/IMG/pdf/3 Bourdoncle 21.pdf

Charland, J.-P. (2000). L'entreprise éducative au Québec, 1840-1900. Québec, QC : Presses de l'Université Laval.

Dubar, C. (2000). La crise des identités : l'interprétation d'une mutation. Paris : Presses universitaires de France. 
Gohier, C. (1997). Éthique et déontologie : l'acte éducatif et la formation des maîtres professionnellement interpellés. Dans M.-P. Desaulniers, F. Jutras, P. Lebuis et G. A. Legault (dir.), Les défis éthiques en éducation (p. 191-205). Québec, QC : Presses de l'Université du Québec.

Gohier, C. (2007). Identités professionnelles d'acteurs de l'enseignement. Regards croisés. Québec, QC : Presses de l'Université du Québec.

Gohier, C. (2013). Éthique et formation : discussion sur les vertus ou vertus de la discussion?. Formation et profession, 21(3), 70-80. http://dx.doi.org/10.18162/fp.2013.210

Gohier, C., Anadón, M., Bouchard, Y., Charbonneau, B. et Chevrier, J. (1999). Vers une vision renouvelée de la professionnalisation de l'enseignement et de la construction de l'identité professionnelle de l'enseignant. Dans C. Gohier, N. Bednarz, L. Gaudreau, R. Pallascio et G. Parent (dir.). L'enseignant, un professionnel (p. 21-56). Québec : Presses de l'Université du Québec.

Hamel, T. (1995). Un siècle de formation des maîtres au Québec : 1836-1939. Montréal, QC : HMH.

Hensler, H. (2004). Pour une ouverture de la culture professionnelle aux savoirs de la recherche en éducation : Quelles conditions aménager en formation initiale et continue?. Dans C. Lessard, M. Altet, L. Paquay et P. Perrenoud, Entre sens commun et sciences bumaines (p. 179-199). Bruxelles : De Boeck Supérieur.

Jeffrey, D. (2013). Profession enseignante : de la moralité exemplaire à léthique professionnelle. Formation et profession, 21(3), 18-29. http://dx.doi.org/10.18162/fp.2013.189

Jeffrey, D. (2014). La moralité vertueuse des enseignantes québécoises. Dans M. Chevrier et C. Froidevaux-Metterie (dir.), Des femmes et des hommes singuliers. Paris : Armand Colin. http://dx.doi.org/10.3917/arco.froid.2014.01.0163

Jeffrey, D. (2015). Enseigner l'éthique aux futurs enseignants. Dans L.-A. St-Vincent (dir.), Le développement de l'agir éthique chez les professionnels en éducation : formations initiale et continue (p. 25-44). Québec : Presses de l'Université du Québec.

Jeffrey, D., Harvengt, D., Vachon, M.-C. et Deschesnes, G. (2008). Le droit et l'éthique dans la profession enseignante. Dans F. Jutras et C. Gohier (dir.), Repères pour l'éthique professionnelle des enseignants (p. 75-92). Québec, QC : Presses de l'Université du Québec.

Lavoie, L. (2001). L'enseignant : un modèle pour la société. Développements récents en droit de l'éducation (vol. 151). Cowansville, QC : Les éditions Yvon Blais.

Legault, G. A. (2003a). Professionnalisme et délibération éthique : manuel d'aide à la décision responsable. Québec, QC : Presses de l'Université du Québec.

Legault, G. A. (dir.) (2003b). Crise d'identité professionnelle et professionnalisme. Québec, QC : Presses de l'Université du Québec.

Lemosse, M. (1989). Le professionnalisme des enseignants : le point de vue anglais. Recherche et formation, (6), 55-66. Repéré à http://ife.ens-lyon.fr/publications/edition-electronique/recherche-et-formation/RR006-05.pdf

Ministère de l'Éducation, du Loisir et du Sport (MELS). (2001). La formation à l'enseignement : les orientations et les compétences professionnelles. Repéré à http://www.education.gouv.qc.ca/fileadmin/site web/documents/reseau/ formation titularisation/formation enseignement orientations EN.pdf

Morin, L. (1997). Postmodernité, éthique et formation des maîtres. Dans M.-P. Desaulniers, F. Jutras, P. Lebuis et G. A. Legault (dir.), Les défis éthiques en éducation (p. 121-149). Québec, QC : Presses de l'Université du Québec.

Parent, A.-M. (1963). Rapport de la Commission royale d'enquête sur l'enseignement dans la province de Québec (5 tomes). Québec, QC : Gouvernement du Québec.

Perrenoud, P. (1994). La formation des enseignants entre théorie et pratique. Paris : L'Harmattan.

Sorel, M., Wittorski, R. (2005). La professionnalisation en actes et en questions. Paris : L'Harmattan.

Schön, D. A. (1994). Le praticien réflexif. À la recherche du savoir caché dans l'agir professionnel. Montréal, QC : Éditions logiques.

Tardif, M. (2005). Résumé de la conférence de Maurice Tardif. Bulletin professionnel Virage, 7(3).

Tardif, M., Lessard, C. et Gauthier C. (1998). Formation des maittres et contextes sociaux : perspectives internationales. Paris : Presses universitaires de France.

Verdier, P. (1999). Morale, éthique, déontologie et droit. Les Cabiers de l'Actif, 276/277, 17-29. Repéré à http://docplayer.fr/3824365-Morale-ethique-deontologie-et-droit.html 


\section{Notes}

1 Avant la Révolution tranquille (1955-1970), le vertuisme des enseignants était promu par le clergé et la bourgeoisie québécoise. Dans les anciennes écoles de formation des maîtres, qui seront remplacées par la formation universitaire dans les années 1960, il était demandé aux futures enseignantes d’être des exemples de moralité. La plus grande partie de leur formation visait à les amener à incarner les vertus catholiques du clergé ultramontain (pudeur, chasteté, pureté du cœur, obéissance à l'autorité).

2 Le rapport Parent, publié en 1963, fait état des orientations politiques prises par la Commission royale d'enquête sur l'enseignement dans la province de Québec formée en 1961. Parmi les apports les plus marquants de ces orientations, soulignons la création d'un ministère de l'Éducation, la scolarisation obligatoire jusqu'à 16 ans, la formation universitaire des enseignants et la création des universités publiques.

3 Il est habituellement accordé et reconnu par un organisme gouvernemental qui détient un tel mandat de l'État. Le Québec s'est doté, à cet égard, de l'Office des professions du Québec.

4 Voir notamment la Fédération des syndicats de l'enseignement : http://fse.qc.net/vie-professionnelle/valorisation-de-la-profession-enseignante/

5 Des enseignantes se plaignent parce que des élèves commencent le préscolaire sans avoir atteint le stade de la propreté. Elles doivent donc agir avec doigté avec ces élèves pour éviter des accusations d'attouchement sexuel. En fait, sont-elles obligées de veiller à l'hygiène d'un élève qui n'a pas atteint le stade de la propreté? D'un point de vue professionnel, cet acte leur appartient-il? Si elles doivent aider un élève lorsqu'il est à la toilette, quel est alors le standard pour cette pratique?

6 Soulignons encore une fois que les recherches savantes et l'expérience des enseignants constituent ce qu'on appelle l'expertise des enseignants.

$7 \quad$ Et le vertuisme catholique ne peut guider l'action dans ce type de situation.

8 Ce phénomène est fort répandu au Québec. En fait, plusieurs enseignants n’ont aucune formation en enseignement. Ils ont été recrutés parce qu'il y a pénurie d'enseignants dans plusieurs champs d'enseignement, notamment ceux qui touchent à la formation professionnelle. Aussi, étant donné la pénurie d'enseignants dans le champ des sciences, des enseignants formés dans les autres disciplines assument cet enseignement. Bref, les champs d'enseignement ne sont pas protégés, par conséquent les enseignants peuvent passer d'un champ à l'autre. Les directions d'établissement considèrent habituellement qu'un enseignant du secondaire peut enseigner toutes les disciplines scolaires, ce qui est tout à fait faux.

9 Soulignons que la standardisation des actes professionnels contribue à la protection et à la réussite des élèves.

10 Au sujet des contacts physiques avec les élèves, une étude réalisée aux États-Unis par Anderson et Levine (1999) a révélé que $41 \%$ des répondants ont affirmé que leur école a institué des protocoles qui limitent les contacts physiques avec les élèves; 61 \% de ces personnes ont confirmé cependant qu'il s'agirait de règles fondées sur des pratiques informelles. Cette même étude fait cas du fait que les enseignants déconseillent fortement à leurs collègues de rester seuls dans une salle avec un élève; cela est fortement déconseillé aux enseignants de sexe masculin à l'égard des élèves du sexe opposé. La majorité des répondants $(67 \%)$ déconseille même de toucher les élèves à l'épaule, peu importe le sexe; une plus large majorité (71\%) déconseille également les gestes d'affection tels que les embrassades ( $h u g$ ) pour montrer de l'affection ou pour mettre l'élève dans une situation plus confortable (p. 837).

11 Cela ne signifie pas qu'il obéit aveuglément à des standards, car il peut expérimenter des nouvelles activités pédagogiques lesquelles seront évaluées à l'usage. Aussi, soulignons que l'invention pédagogique demande de longues années d'expérience, un enseignant ne peut improviser une pratique sans suivre à cet égard un protocole de recherche.

\section{Pour citer cet article}

Jeffrey, D. (2016). L'éthique et la standardisation des pratiques enseignantes. Formation et profession, 24(2), 5-16. http://dx.doi.org/10.18162/fp.2016.351 